\title{
The co-mutation of EGFR and tumor-related genes leads to a worse prognosis and a higher level of tumor mutational burden in Chinese non-small cell lung cancer patients
}

\author{
Yi Zhang ${ }^{1 \#}$, Shirong $\mathrm{Li}^{2 \#}$, Zhi Lyu ${ }^{3 \#}$, Jinghuang Cai ${ }^{4}$, Naishan Zheng ${ }^{5}$, Yanping $\mathrm{Li}^{5}$, Tianwen $\mathrm{Xu}^{6}$, \\ Huiqing Zeng ${ }^{4}$
}

${ }^{1}$ Department of Cardiothoracic Surgery, Zhangzhou Hospital Affiliated of Fujian Medical University, Zhangzhou, China; ${ }^{2}$ Department of Respiratory, Wuhu Hospital Affiliated to East China Normal University, Wuhu, China; ${ }^{3}$ Department of Senior Cadres Ward, Zhongshan Hospital of Xiamen University, School of Medicine, Xiamen University, Xiamen, China; ${ }^{4}$ Department of Pulmonary and Critical Care Medicine, Zhongshan Hospital of Xiamen University, School of Medicine, Xiamen University, Xiamen, China; ${ }^{5}$ The Third Clinical Medical College, Fujian Medical University, Fuzhou, China; ${ }^{6}$ Department of Medical Oncology, the Second Affiliated Hospital of Fujian Medical University, Quanzhou, China Contributions: (I) Conception and design: H Zeng, T Xu, Y Zhang, S Li, Z Lyu; (II) Administrative support: Y Li, T Xu; (III) Provision of study materials or patients: Z Lyu, Y Cai, N Zheng; (IV) Collection and assembly of data: Y Zhang, S Li, J Cai, Y Li; (V) Data analysis and interpretation: Y Zhang, S Li, Z Lyu; (VI) Manuscript writing: All authors; (VII) Final approval of manuscript: All authors.

\#These authors contributed equally to this work.

Correspondence to: Huiqing Zeng. Department of Respiratory and Critical Care Medicine, Zhongshan Hospital, Xiamen University, 205 Hubin South Road, Xiamen 361004, China. Email: zhq20071212@xmu.edu.cn; Tianwen Xu. The Second Affiliated Hospital of Fujian Medical University, No. 34, Zhongshan North Road, Licheng District, Quanzhou, China. Email: Xutianwen53@163.com.

Background: Lung cancer is the leading cause of cancer mortality in China. The clinicopathologic features and genetic profile of Chinese lung cancer patients need to be investigated. This study evaluated the gene mutation profile, analyzed the frequency of concurrent genes in epidermal growth factor receptor (EGFR)mutant non-small cell lung cancer (NSCLC) patients, and determined its prognostic value.

Methods: We collected the clinical data from 151 initially diagnosed patients NSCLC. Tumor samples underwent targeted next-generation sequencing (NGS). Progression-free survival (PFS) and overall survival (OS) were analyzed using the Kaplan-Meier method.

Results: Among the 151 participants, the mutational profile revealed alterations in 29 genes, where TP53 (37.09\%) and EGFR (30.46\%) exhibited the highest mutation rates. Mutations in the EGFR gene were most prevalent (40\%) in adenocarcinoma (LUAD) and were only present in $8.8 \%$ of participants with squamous cell carcinoma (LUSC). The most frequently mutated genes in LUAD patients were TP53 (47\%), followed by KRAS (11.7\%). In all 39 participants with EGFR mutations, TP53, KRAS, PIK3CA, APC, and $F B X W 7$ were also mutated. Those with only EGFR mutation appeared to have a better prognosis; however, the difference was not statistically significant. Tumor mutational burden (TMB) was roughly significantly increased in patients who harbored EGFR and other mutant driver genes, compared with only EGFR mutant patients. The TMB value was significantly higher in those with P53 mutation than in P53 wild-type patients. Conclusions: We described the genetic profiles of NSCLC and compared the difference in genetic profiles between LUAD and LUSC. The concomitant genetic alterations might be a poor prognostic factor for patients with EGFR mutation, and TMB might be prognostically related to the co-mutations of EGFR and other genes.

Keywords: Non-small cell lung cancer (NSCLC); tumor mutational burden (TMB); epidermal growth factor receptor $(E G F R)$; prognosis

Submitted Sep 16, 2021. Accepted for publication Jan 17, 2022.

doi: 10.21037/jtd-21-1921

View this article at: https://dx.doi.org/10.21037/jtd-21-1921

(c) Journal of Thoracic Disease. All rights reserved. 


\section{Introduction}

Lung cancer is the main cause of cancer deaths globally and in China (1). Non-small cell lung cancer (NSCLC) is the most common type of lung cancer, comprising approximately $80 \%(2,3)$. Adenocarcinoma (about half of cases) and Squamous cell carcinoma (about a quarter of cases) are the main histological subtypes of NSCLC (4). The treatment of NSCLC includes chemoradiotherapy, surgery, targeted therapy, and immunotherapy (5).

With the accumulation of mutations, if the key genes that control cell survival and proliferation undergo activating mutations, their continuous activation of signal pathways related to cell proliferation will lead to malignant transformation of cells and obtain the characteristics of a series of malignant tumors such as immortal proliferation and metastasis $(6,7)$, so this type of mutation is also called a driver mutation (8). Previous study demonstrated that nextgeneration sequencing (NGS) technologies were performed to identify the genetic characterizations of NSCLC and determine targets of tyrosine kinase inhibitors (TKIs) (9). The most frequent somatic changes were detected in certain genes, such as EGFR, TP53, FGFR1, KRAS, PIK3CA, ERBB2 (HER2), BRAF, ALK, ROS1, MAP2K1/MEK1, RET, $N R A S$, and $A K T 1$ (10).

Each histological subtype of cancer has its own unique genetic mutational profile, based on different genetic and environmental risk factors. Genetic profiling of the individual tumor could facilitate understanding of the distinct molecular mechanism that regulates cancer progression and reveal potential therapeutic targets. Lung adenocarcinoma (LUAD) and squamous cell carcinoma (LUSC) should have different genetic profiles, responsible for their different responses to therapies.

Epidermal growth factor receptor $(E G F R)$ and tumor suppressor TP53 genes have been shown to be the 2 most commonly mutated genes in patients with NSCLC. Deletion of EGFR exon19 and exon21 L858R represent 85$90 \%$ EGFR activation mutations in lung cancer, and they are sensitive to approved EGFR-TKIs such as erlotinib, gefitinib, and afatinib (11-13). Exon18 and 20 mutations are uncommon mutations and less sensitive or completely insensitive to TKIs (14). The tumor protein p53 (TP53) is a tumor suppressor gene and the most commonly mutated gene in human cancers (15). Patients who carry TP53 mutations generally might experience more aggressive disease, increased rates of resistance to chemotherapy, and shorter survival $(16,17)$. It has been reported that patients with both EGFR and TP53 mutations might have decreased responsiveness to EGFR-TKIs $(18,19)$. Barnet et al. reported that PIK3CA and EGFR co-mutation provide clinically meaningful information predicting a poor response (20). This study analyzed the prognostic implication of co-mutations of other cancer-related genes in LUAD patients with EGFR mutation.

Tumor mutational burden (TMB) is a hallmark of cancer, which has been associated with microsatellite instability, defective DNA replication/repair, and response to PD-1 and PD-L1 blockade immunotherapy (21). The TMB can be calculated from somatic mutation counts in the coding genome using NGS data. The determination of TMB is of great value not only in the characteristics of tumors but also in predicting the clinical efficacy of immunotherapy. Elevated TMB has been related to the efficacy of immune checkpoint blockade (ICB) response in lung cancers and other tumor types (22-24).

Our study aimed to determine the clinicopathologic features and genetic profile of Chinese NSCLC patients. We attempted to elucidate the prognostic impact of comutations in other cancer-related genes on survival in patients with NSCLC harboring EGFR mutations. We present the following article in accordance with the REMARK reporting checklist (available at https://jtd. amegroups.com/article/view/10.21037/jtd-21-1921/rc).

\section{Methods}

\section{Patients and samples}

Matched pairs of tumor and normal tissue samples of 151 Chinese patients with NSCLC were collected in Zhongshan Hospital of Xiamen University. Basic and clinicopathological information from all participants was included and all samples were formalin-fixed and paraffin-embedded. This study was approved by the Ethics Committee of Zhongshan Hospital of Xiamen University (No. 2021-172). All procedures performed in this study involving human participants were under the Declaration of Helsinki (revised in 2013). Written informed consent was obtained from all study participants. We extracted DNA using a formalin-fixed paraffin-embedded whole-genome extraction kit (cat. No. 180134, Qiagen, Hilden, Germany) according to the manufacturer's protocol.

\section{Targeted NGS}

The DNeasy Blood \& Tissue Kit (69504, QIAGEN, Venlo, Netherlands) was used to extract total DNA by strictly 
Table 1 Basic clinical characteristics of lung cancer patients

\begin{tabular}{|c|c|}
\hline Characteristics & $N=151$ \\
\hline Age (years) & $65.76 \pm 10.74$ \\
\hline \multicolumn{2}{|l|}{ Gender (\%) } \\
\hline Male & 64.24 \\
\hline Female & 35.76 \\
\hline \multicolumn{2}{|l|}{ Histology (\%) } \\
\hline Adenocarcinoma & 65.56 \\
\hline Squamous cell carcinoma & 22.52 \\
\hline Unclear & 11.92 \\
\hline \multicolumn{2}{|l|}{ Stage (\%) } \\
\hline 1 & 0.66 \\
\hline 2 & 3.31 \\
\hline 3 & 17.22 \\
\hline 4 & 65.56 \\
\hline Unknown & 13.25 \\
\hline Smoking history (\%) & $55.41(82 / 148)$ \\
\hline \multicolumn{2}{|l|}{ Metastasis (\%) } \\
\hline Brain metastasis & $2.95(13 / 132)$ \\
\hline Bone metastasis & $9.84(39 / 132)$ \\
\hline
\end{tabular}

follow the procedures. Agilent's Bioanalyzer (Agilent Technologies, Santa Clara, CA, USA) was used to assess the amount of DNA. The NGS was performed by five different lung cancer-related gene panels and whole-exome sequencing (WES).

\section{Mutation calling and analysis}

The sequencing data were compared and sequenced by Burrow-Wheeler Aligner (BWA) (25) with the human reference genome (NCBI Build 37; https://www.ncbi.nlm. nih.gov/genome/guide/human/). The duplicate polymerase chain reaction (PCR) data was removed through SAMtools (http://www.htslib.org/) (26). Vardict is based on the compilation and detection algorithm of heuristic algorithm to realize the mutation extraction of SNP and INDEL, and judge the authenticity of the site through mutation reads and database information. Annovar can determine whether SNP or $\mathrm{CNV}$ causes protein coding changes and affected amino acids, and can autonomously select gene definition systems (such as RefSeq/UCSC/Ensemble, etc.), and can also identify variants of specific genomic regions, or link other databases to variants Make comments (e.g., dbSNP/1000 Genome Project). The variation call files generated by VarDict (27) were exegesis with ANNOVAR (28) and then modified to MAF files using maftools (29). After removing all synonymous mutations from the MAF file, maftools was used to calculate the variance of each participant. Only non-synonymous mutations were included in the TMB (mutations/MB) calculation.

\section{Statistical analysis}

Continuous variables were expressed as medians and interquartile ranges (IQR) and categorical variables were presented as percentages, using analysis of variance (ANOVA) test and chi-square test for comparison of their differences, respectively. These statistical analyses were performed by SPSS version 26.0 (IBM Corp., Chicago, IL, USA). Overall survival (OS) was analyzed using the KaplanMeier method and the log-rank test was used to compare survival curves, which were performed by Graphpad Prism version 8 (GraphPad Software, San Diego, CA, USA).

\section{Results}

\section{Clinical characteristics}

A total of 151 NSCLC samples underwent molecular testing at initial diagnosis, among which 142 were detected by different cancer-related gene panels, and the others were detected by WES. The cohort average age was $65.76 \pm 10.74$ years. There were $97(97 / 151,64.24 \%)$ male and $54(54 / 151,35.76 \%)$ female patients. There were $99(99 / 151,65.56 \%)$ cases of LUAD and 34 (34/151, $22.52 \%)$ cases of LUSC. Slightly more than half of the participants $(82 / 148,55.41 \%)$ had a history of smoking. Most participants had stage III $(17.22 \%, \mathrm{n}=26)$ or IV $(65.56 \%, \mathrm{n}=99)$ disease at the time of initial diagnosis; $9.84 \%$ (13/132) patients had brain metastasis, and 2.95\% (39/132) of patients had bone metastasis (Table 1).

The distribution of mutated genes is shown in Figure 1 A. The top three frequent mutated genes were TP53, $E G F R$, and KRAS. We found a distinct difference of mutation pattern between LUAD and LUSC from the somatic mutational profile of the patients. As shown in Figure 1B,1C, EGFR mutations were most prevalent in LUAD, and approximately $40 \%$ of participants harbored somatic EGFR mutations. Unlike LUAD, only $8.8 \%$ of LUSC patients carried somatic mutations of EGFR. The 

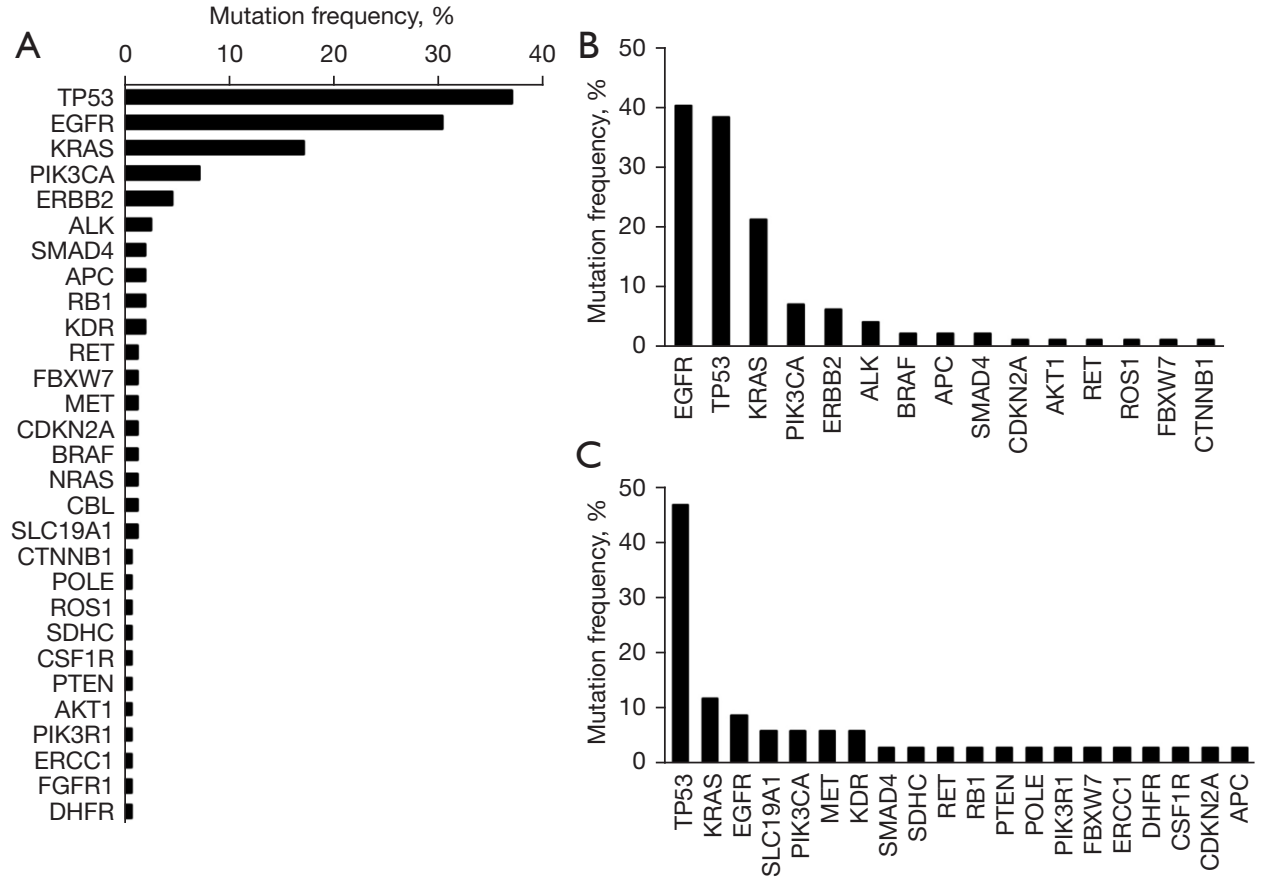

Figure 1 Mutational profiling of Chinese NSCLC patients. (A) Genetic alteration frequency identified by NGS in all 151 samples; (B,C) genetic alteration frequency identified by NGS in LUAD (B) and LUSC (C) patients. NSCLC, non-small-cell lung cancer; NGS, nextgeneration sequencing; LUAD, lung adenocarcinoma; LUSC, lung squamous cell carcinoma.

most frequent mutant genes in LUSC participants were TP53 (47\%), followed by KRAS (11.7\%).

There were 39 LUAD and 3 LUSC participants identified with somatic EGFR mutations. Of these NSCLC cases, 85.7\% (36/42) patients [84.6\% (33/39) LUAD and $100 \%(3 / 3)$ LUSC patients] had only one type EGFR mutation, while the other $14.3 \%$ had compound $E G F R$ mutations (with 2 or more EGFR mutations). Due to the low incidence of EGFR in LUSC patients (only three patients), we show the statistical results of EGFR mutation types and mutation frequencies in LUAD patients in Figure 2. The patients with only EGFR mutations were distributed in exon18 (2.6\%), exon19 (30.8\%), exon20 (12.8\%), and exon21 (38.4\%). Compound EGFR mutations were located in exon19 del + CNV (5.1\%), L858R + CNV (5.1\%), $\mathrm{G} 719 \mathrm{C}+\mathrm{p} . \mathrm{S} 768 \mathrm{I}+\mathrm{CNV}(2.6 \%)$, and $\mathrm{E} 709 \mathrm{~K}+$ p.L858R $(2.6 \%)$.

\section{Prognostic implications of co-mutations with EGFR mutation in LUAD patients}

Among the 39 EGFR-mutated LUAD patients, 20 patients were concomitant to other somatic cancer-related gene mutations; that is, the incidence of cancer-related gene co-mutation in patients with lung adenocarcinoma in NSCLC was 20.2\% (20/99). Mutations occurring alongside EGFR mutations included those of TP53, KRAS, PIK3CA, $A P C$, and $F B X W 7$. Among these 5 genes, TP53 was the most frequently co-mutated (Figure $3 A$ ). We analyzed the prognostic implication of co-mutations of cancerrelated genes in LUAD patients with EGFR mutation by comparing the OS and progression-free survival (PFS) between the two groups (Figure $3 B-3 E$ ). The participants with $E G F R$ mutation only appeared to have a better prognosis (Figure 3B,3C); however, the difference was not statistically significant ( $\mathrm{P}=0.25$ for $\mathrm{PFS} ; \mathrm{P}=0.34$ for $\mathrm{OS}$ ). Among participants with co-mutations of EGFR and other genes, there were 17 patients with TP53 gene mutations (Figure 3D,3E); however, there was no significant difference in the prognosis between participants with EGFR and TP53 co-mutation and those with only EGFR mutation.

\section{TMB of LUAD patients with EGFR mutations}

The TMB values were calculated from the mutation data of 23 LUAD patients. As shown in Figure 4A, among the 10 


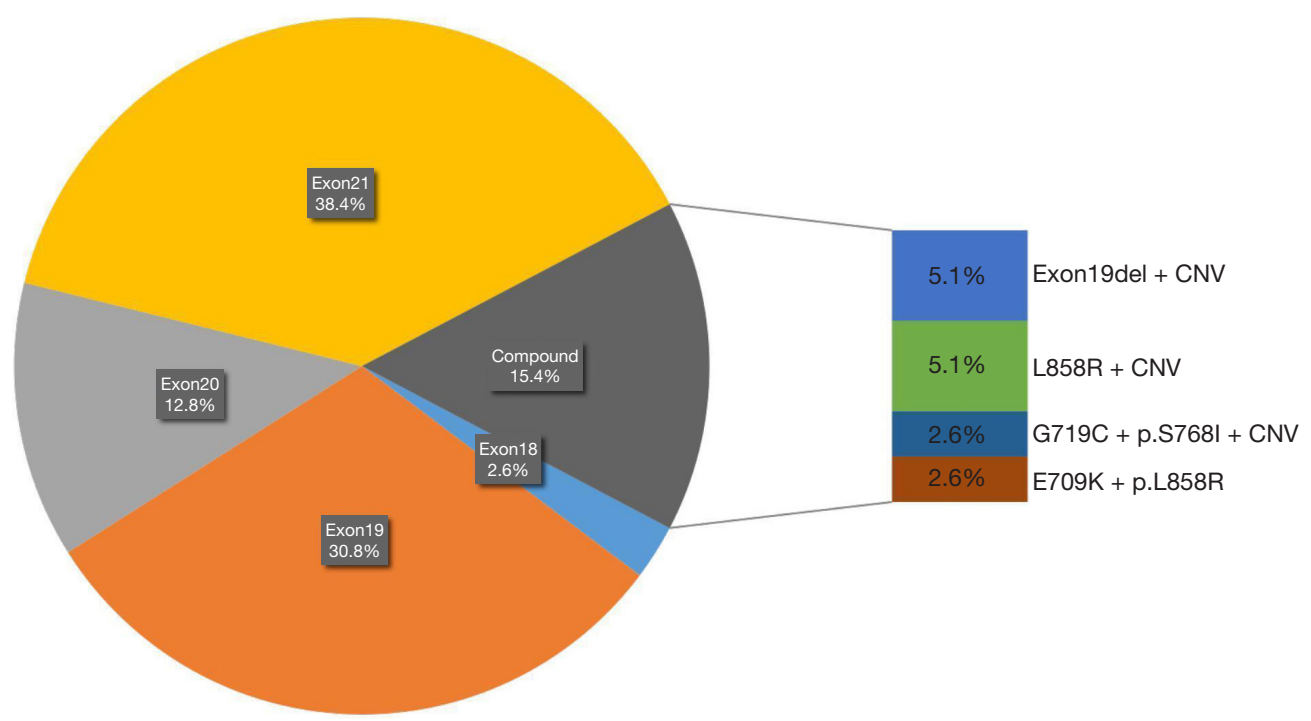

Figure 2 Frequency and distribution of EGFR mutations in LUAD patients. EGFR, epidermal growth factor receptor; LUAD, lung adenocarcinoma.

of 23 LUAD participants with EGFR mutation, the TMB value was not significant difference between $E G F R$ wildtype and EGFR mutation patients $(\mathrm{P}=0.57)$. The TMB value was roughly significantly increased in patients who harbored EGFR and other driver genes co-mutations, compared with only EGFR mutant patients (Figure $4 B$, $\mathrm{P}=0.14$ ). Furthermore, the TMB value was significantly higher in participants with $P 53$ mutation than in $P 53$ wildtype participants (Figure $4 C, \mathrm{P}=0.002$ ). However, in the patients with $E G F R$ mutation, the TMB values were not significantly different between $P 53$ mutation and $P 53$ wildtype (Figure $4 D, \mathrm{P}=0.25$ ).

\section{Discussion}

Lung cancer is the leading cause of death among men and the second leading cause among women. A previous study revealed the differences in genetic profiles of tumor tissues between LUAD and LUSC: Ding et al. analyzed the mutational profile of 318 Chinese NSCLC patients and found that more than half of LUAD patients had EGFR mutation, and a total of $90 \%$ of the LUSC patients carried the TP53 gene mutation (30). In this study, we described the somatic mutation profile of NGS in Chinese NSCLC patients, assessed the impact of co-mutation of EGFR and other cancer-related genes on the prognosis, and the differences in TMB between these groups. We found that $E G F R$ is the most frequently mutated gene in LUAD patients. The most commonly mutated gene in LUSC patients is TP53. Patients with LUAD who carry EGFR mutations and without other cancer-related gene mutations seem to have longer PFS and OS. In patients with EGFR mutations, TP53 wild-type and TP53 mutation do not affect the prognosis. We found that the TMB of patients with $E G F R$ single mutation was significantly lower than patients with co-mutation of $E G F R$ and cancer-related genes.

Almost all of the driver mutations currently found in non-small cell lung cancer occur in key kinases that control cell survival and proliferation signaling pathways, including members of the receptor tyrosine family expressed on cell membranes (such as EGFR, HER2, FGFR, DDR2, and $M E T$ ), as well as some kinases in the cell membrane (such as KRAS, BRAF, AKT1, P13KCA, and MEK1) (31,32). Some previous studies have suggested that the differences in prognosis for NSCLC patients was due to mutations in EGFR. For instance, clinical data has shown that the benefit of EGFR-TKIs was greater for patients with exon19 deletions than exon21 L858R substitutions $(33,34)$. Patients with exon21 L858R substitutions had significantly longer PFS compared to those with exon19 deletions who underwent chemotherapy (35). The differences in cooccurring molecular events may partly explain the clinical heterogeneity in patients with EGFR-mutant NSCLC. Tang et al. reported several types of research about the prognosis of EGFR-mutant patients with concomitant genetic alterations (36). In our study, the top co-mutated 

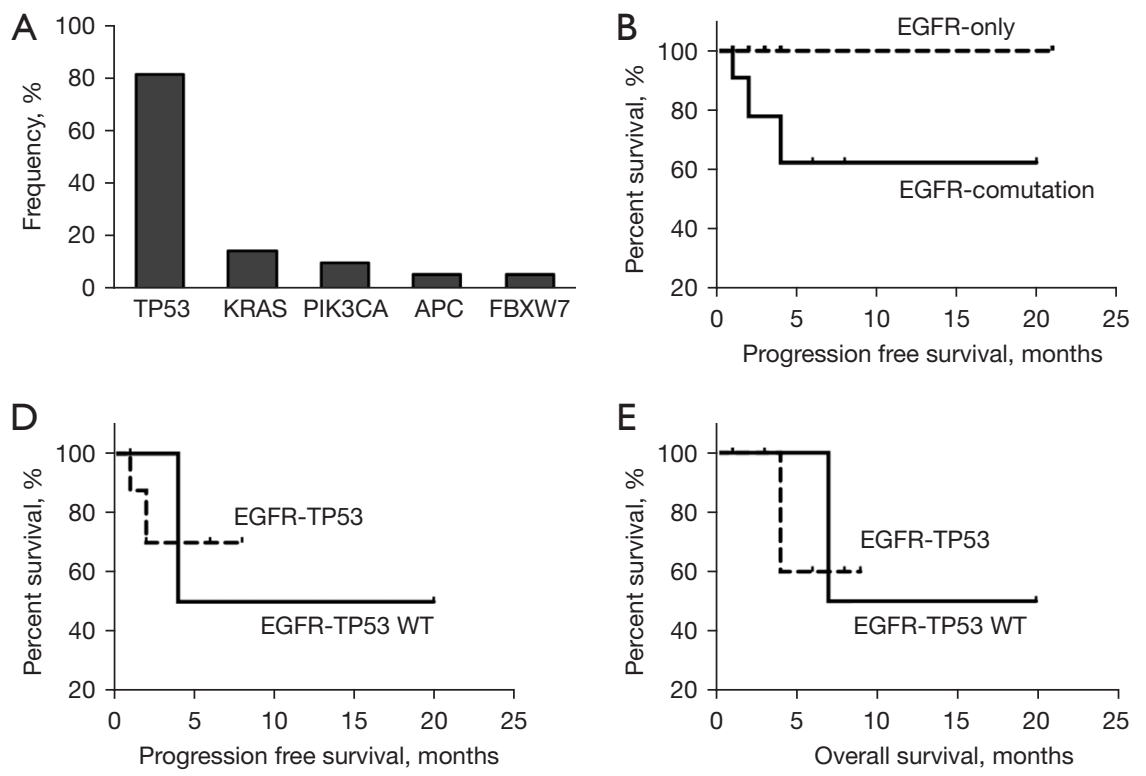

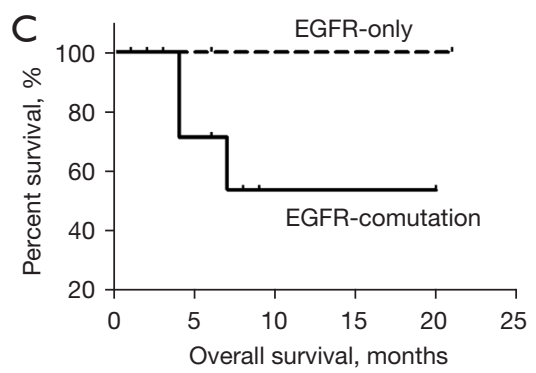

Figure 3 The differences of prognosis between single EGFR mutation and concurrent gene alterations patients. (A) Co-occurring genetic alterations of LUAD EGFR-mutant patients. (B,C) Kaplan-Meier analyses of PFS (B, HR 0.20; 95\% CI: 0.019-2.17) and OS (C, HR 0.25; 95\% CI: 0.014-4.43), respectively, between EGFR-only mutation and co-mutation with other genes. (D,E) Kaplan-Meier estimates the differences of progression-free survival (D, HR 1.07; 95\% CI: 0.09-12.31) and overall survival (E, HR 0.89; 95\% CI: 0.07-11.36) between EGFR and TP53 co-mutation patients and EGFR mutation with TP53 wild-type patients. EGFR, epidermal growth factor receptor; LUAD, lung adenocarcinoma; PFS, progression-free survival; OS, overall survival; HR, hazard ratio; CI, confidence interval.
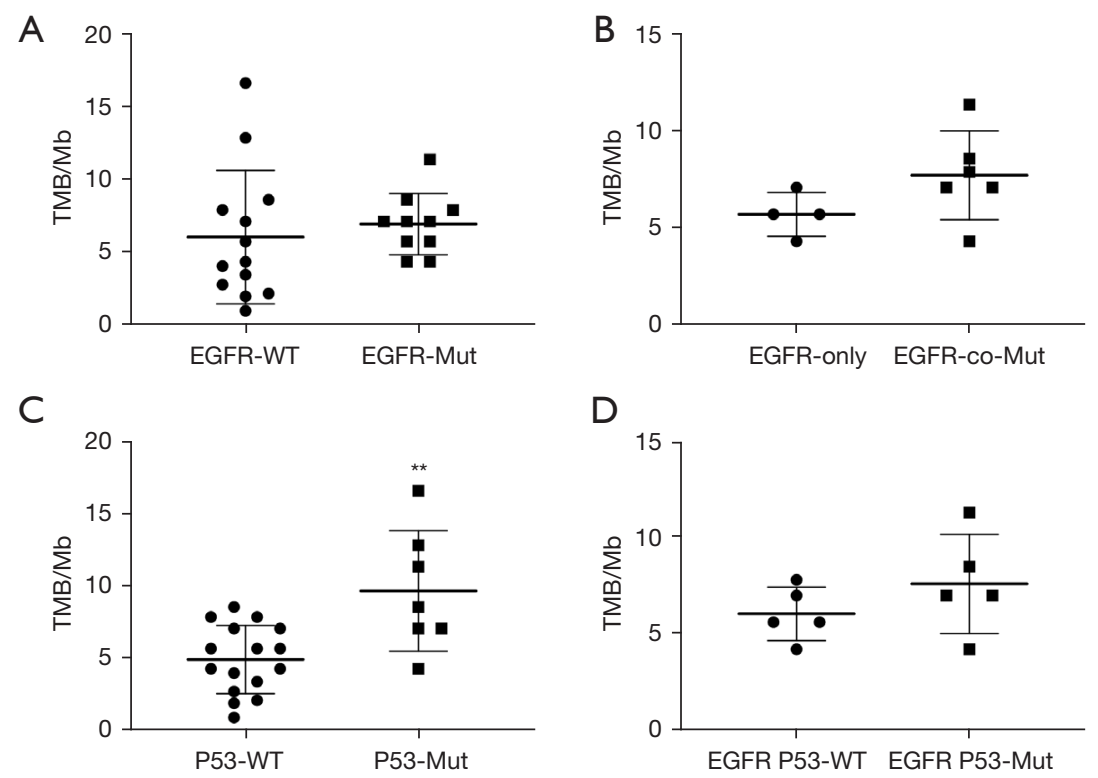

Figure 4 The TMB values of LUAD patients. (A) EGFR-mutant versus EGFR wild-type patients; (B) EGFR-Only mutant versus comutant patients. (C) P53 wild-type vs. P53 mutant patients. (D) EGFR and P53 co-mutation vs. EGFR mutation with TP53 wild-type patients. ${ }^{* *}, 0.001<\mathrm{P}<0.01$. TMB, tumor mutational burden; EGFR, epidermal growth factor receptor. 
genes with EGFR in NSCLC patients were TP53, KRAS, and PIK3CA. Eng et al. showed that concurrent PIK3CA mutation did not decrease the efficacy of EGFR-TKIs in patients who had EGFR/PIK3CA co-mutation (37). In a Chinese cohort, NSCLC patients without co-mutation genes had a significantly longer PFS with EGFR-TKIs treatment and no significant OS difference between patients with single EGFR mutation and co-mutation genes (38). In our study, the patients with EGFR mutation only seemed to have longer PFS and OS, but without statistical significance, which might be due to the small sample size.

Multiple somatic mutations in the same tumor are associated with poorer outcomes in resected NSCLC (39). Hence, several studies have focused on the prognostic effect of co-mutated TP53 and EGFR genes. Labbe et al. demonstrated that the coexistence of a TP53 mutation had no influence on relapse-free survival (RFS) or OS after primary surgical resection, and PFS while on first-line EGFR-TKI therapy was significantly shorter for patients with dual mutations when comparing only TP53 missense mutations to TP53 wild-type (40). Jiao et al. reported that TP53 mutation was not conducive to OS in metastatic NSCLC patients, and the estimated survival time for wildtype TP53 was significantly longer than mutated TP53 (41). In this study, the prognosis was no different between TP53 WT and TP53 mutant patients who carried EGFR mutation. Maybe because the participants were divided into subgroups according to different treatment strategies, the number of participants that underwent prognosis assessment was limited.

A previous study revealed that the TMB was lower in EGFR mutant patients than EGFR wild-type patients in lung cancer, which may play a negative role in the efficacy of EGFR-TKI therapy in lung cancer patients who carry the EGFR-mutant (42). In our study, we found that TMB was significantly lower in EGFR only mutation participants than those with EGFR co-mutation. We speculate that TMB is a helpful marker for predicting the efficacy of targeted therapy. However, whether TMB is associated with clinical outcomes remains to be elucidated.

\section{Conclusions}

This study revealed the genetic profiles of NSCLC and compared the difference in genetic profiles between LUAD and LUSC. Concomitant genetic alterations might be a poor prognostic factor for patients with EGFR mutation, and TMB might be related to the co-mutations of EGFR and other genes in terms of prognosis.

\section{Acknowledgments}

The authors are grateful to Shanghai Tongshu Biotechnology Co., Ltd. for technical support.

Funding: None.

\section{Footnote}

Reporting Checklist: The authors have completed the REMARK reporting checklist. Available at https://jtd. amegroups.com/article/view/10.21037/jtd-21-1921/rc

Data Sharing Statement: Available at https://jtd.amegroups. com/article/view/10.21037/jtd-21-1921/dss

Conflicts of Interest: All authors have completed the ICMJE uniform disclosure form (available at https://jtd.amegroups. com/article/view/10.21037/jtd-21-1921/coif). All authors report the technical support for this study from Shanghai Tongshu Biotechnology Co., Ltd. The authors have no other conflicts of interest to declare.

Ethical Statement: The authors are accountable for all aspects of the work in ensuring that questions related to the accuracy or integrity of any part of the work are appropriately investigated and resolved. This study was approved by the Ethics Committee of Zhongshan Hospital of Xiamen University (No. 2021-172). All procedures performed in this study involving human participants were under the Declaration of Helsinki (revised in 2013). Written informed consent was obtained from all study participants.

Open Access Statement: This is an Open Access article distributed in accordance with the Creative Commons Attribution-NonCommercial-NoDerivs 4.0 International License (CC BY-NC-ND 4.0), which permits the noncommercial replication and distribution of the article with the strict proviso that no changes or edits are made and the original work is properly cited (including links to both the formal publication through the relevant DOI and the license). See: https://creativecommons.org/licenses/by-nc-nd/4.0/.

\section{References}

1. Global Burden of Disease Cancer Collaboration; Fitzmaurice C, Allen C, et al. Global, Regional, and 
National Cancer Incidence, Mortality, Years of Life Lost, Years Lived With Disability, and Disability-Adjusted Lifeyears for 32 Cancer Groups, 1990 to 2015: A Systematic Analysis for the Global Burden of Disease Study. JAMA Oncol 2017;3:524-48.

2. Lortet-Tieulent J, Soerjomataram I, Ferlay J, et al. International trends in lung cancer incidence by histological subtype: adenocarcinoma stabilizing in men but still increasing in women. Lung Cancer 2014;84:13-22.

3. Pirker R, Filipits M. Personalized treatment of advanced non-small-cell lung cancer in routine clinical practice. Cancer Metastasis Rev 2016;35:141-50.

4. Osmani L, Askin F, Gabrielson E, et al. Current WHO guidelines and the critical role of immunohistochemical markers in the subclassification of non-small cell lung carcinoma (NSCLC): Moving from targeted therapy to immunotherapy. Semin Cancer Biol 2018;52:103-9.

5. Soo RA, Stone ECA, Cummings KM, et al. Scientific Advances in Thoracic Oncology 2016. J Thorac Oncol 2017;12:1183-209.

6. Weinstein IB, Joe A. Oncogene addiction. Cancer Res 2008;68:3077-80; discussion 3080.

7. Mehnert JM, Kluger HM. Driver mutations in melanoma: lessons learned from bench-to-bedside studies. Curr Oncol Rep 2012;14:449-57.

8. De S, Ganesan S. Looking beyond drivers and passengers in cancer genome sequencing data. Ann Oncol 2017;28:938-45.

9. Pao W, Girard N. New driver mutations in non-small-cell lung cancer. Lancet Oncol 2011;12:175-80.

10. Levy MA, Lovly CM, Pao W. Translating genomic information into clinical medicine: lung cancer as a paradigm. Genome Res 2012;22:2101-8.

11. Mok TS, Wu YL, Thongprasert S, et al. Gefitinib or carboplatin-paclitaxel in pulmonary adenocarcinoma. $\mathrm{N}$ Engl J Med 2009;361:947-57.

12. Rosell R, Carcereny E, Gervais R, et al. Erlotinib versus standard chemotherapy as first-line treatment for European patients with advanced EGFR mutation-positive non-small-cell lung cancer (EURTAC): a multicentre, open-label, randomised phase 3 trial. Lancet Oncol 2012;13:239-46.

13. Sequist LV, Yang JC, Yamamoto N, et al. Phase III study of afatinib or cisplatin plus pemetrexed in patients with metastatic lung adenocarcinoma with EGFR mutations. J Clin Oncol 2013;31:3327-34.

14. Kobayashi Y, Mitsudomi T. Not all epidermal growth factor receptor mutations in lung cancer are created equal:
Perspectives for individualized treatment strategy. Cancer Sci 2016;107:1179-86.

15. Hollstein M, Sidransky D, Vogelstein B, et al. p53 mutations in human cancers. Science 1991;253:49-53.

16. Tsao MS, Aviel-Ronen S, Ding K, et al. Prognostic and predictive importance of $\mathrm{p} 53$ and RAS for adjuvant chemotherapy in non small-cell lung cancer. J Clin Oncol 2007;25:5240-7.

17. Custodio AB, González-Larriba JL, Bobokova J, et al. Prognostic and predictive markers of benefit from adjuvant chemotherapy in early-stage non-small cell lung cancer. J Thorac Oncol 2009;4:891-910.

18. VanderLaan PA, Rangachari D, Mockus SM, et al. Mutations in TP53, PIK3CA, PTEN and other genes in EGFR mutated lung cancers: Correlation with clinical outcomes. Lung Cancer 2017;106:17-21.

19. Molina-Vila MA, Bertran-Alamillo J, Gascó A, et al. Nondisruptive p53 mutations are associated with shorter survival in patients with advanced non-small cell lung cancer. Clin Cancer Res 2014;20:4647-59.

20. Barnet MB, O'Toole S, Horvath LG, et al. EGFRCo-Mutated Advanced NSCLC and Response to EGFR Tyrosine Kinase Inhibitors. J Thorac Oncol 2017;12:585-90.

21. Roberts SA, Gordenin DA. Hypermutation in human cancer genomes: footprints and mechanisms. Nat Rev Cancer 2014;14:786-800.

22. Rizvi NA, Hellmann MD, Snyder A, et al. Cancer immunology. Mutational landscape determines sensitivity to PD-1 blockade in non-small cell lung cancer. Science 2015;348:124-8.

23. Snyder A, Makarov V, Merghoub T, et al. Genetic basis for clinical response to CTLA-4 blockade in melanoma. N Engl J Med 2014;371:2189-99.

24. Schumacher TN, Schreiber RD. Neoantigens in cancer immunotherapy. Science 2015;348:69-74.

25. Slater PM, Grivell R, Cyna AM. Labour management of a woman with carnitine palmitoyl transferase type 2 deficiency. Anaesth Intensive Care 2009;37:305-8.

26. Li H. A statistical framework for SNP calling, mutation discovery, association mapping and population genetical parameter estimation from sequencing data. Bioinformatics 2011;27:2987-93.

27. Lai $Z$, Markovets A, Ahdesmaki M, et al. VarDict: a novel and versatile variant caller for next-generation sequencing in cancer research. Nucleic Acids Res 2016;44:e108.

28. Wang K, Li M, Hakonarson H. ANNOVAR: functional annotation of genetic variants from high-throughput 
sequencing data. Nucleic Acids Res 2010;38:e164.

29. Mayakonda A, Lin DC, Assenov Y, et al. Maftools: efficient and comprehensive analysis of somatic variants in cancer. Genome Res 2018;28:1747-56.

30. Ding Y, Zhang L, Guo L, et al. Comparative study on the mutational profile of adenocarcinoma and squamous cell carcinoma predominant histologic subtypes in Chinese non-small cell lung cancer patients. Thorac Cancer 2020;11:103-12.

31. Sankar K, Gadgeel SM, Qin A. Molecular therapeutic targets in non-small cell lung cancer. Expert Rev Anticancer Ther 2020;20:647-61.

32. Chen JA, Riess JW. Optimal Management of Patients with Advanced NSCLC Harboring High PD-L1 Expression and Driver Mutations. Curr Treat Options Oncol 2020;21:60.

33. Lee CK, Wu YL, Ding PN, et al. Impact of Specific Epidermal Growth Factor Receptor (EGFR) Mutations and Clinical Characteristics on Outcomes After Treatment With EGFR Tyrosine Kinase Inhibitors Versus Chemotherapy in EGFR-Mutant Lung Cancer: A MetaAnalysis. J Clin Oncol 2015;33:1958-65.

34. Yang JC, Wu YL, Schuler M, et al. Afatinib versus cisplatin-based chemotherapy for EGFR mutation-positive lung adenocarcinoma (LUX-Lung 3 and LUX-Lung 6): analysis of overall survival data from two randomised, phase 3 trials. Lancet Oncol 2015;16:141-51.

35. Ke EE, Wu YL. Afatinib in the first-line treatment of epidermal-growth-factor-receptor mutation-positive nonsmall cell lung cancer: a review of the clinical evidence. Ther Adv Respir Dis 2016;10:256-64.

Cite this article as: Zhang Y, Li S, Lyu Z, Cai J, Zheng N, Li Y, Xu T, Zeng H. The co-mutation of EGFR and tumorrelated genes leads to a worse prognosis and a higher level of tumor mutational burden in Chinese non-small cell lung cancer patients. J Thorac Dis 2022;14(1):185-193. doi: 10.21037/jtd21-1921
36. Tang Y, Che N, Yu Y, et al. Co-occurring genetic alterations and primary EGFR T790M mutations detected by NGS in pre-TKI-treated NSCLCs. J Cancer Res Clin Oncol 2020;146:407-16.

37. Eng J, Woo KM, Sima CS, et al. Impact of Concurrent PIK3CA Mutations on Response to EGFR Tyrosine Kinase Inhibition in EGFR-Mutant Lung Cancers and on Prognosis in Oncogene-Driven Lung Adenocarcinomas. J Thorac Oncol 2015;10:1713-9.

38. Hu W, Liu Y, Chen J. Concurrent gene alterations with EGFR mutation and treatment efficacy of EGFR-TKIs in Chinese patients with non-small cell lung cancer. Oncotarget 2017;8:25046-54.

39. Tamiya A, Koh Y, Isa SI, et al. Impact of somatic mutations on prognosis in resected non-small-cell lung cancer: The Japan Molecular Epidemiology for lung cancer study. Cancer Med 2020;9:2343-51.

40. Labbé C, Cabanero M, Korpanty GJ, et al. Prognostic and predictive effects of TP53 co-mutation in patients with EGFR-mutated non-small cell lung cancer (NSCLC). Lung Cancer 2017;111:23-9.

41. Jiao XD, Qin BD, You P, et al. The prognostic value of TP53 and its correlation with EGFR mutation in advanced non-small cell lung cancer, an analysis based on cBioPortal data base. Lung Cancer 2018;123:70-5.

42. Offin M, Rizvi H, Tenet M, et al. Tumor Mutation Burden and Efficacy of EGFR-Tyrosine Kinase Inhibitors in Patients with EGFR-Mutant Lung Cancers. Clin Cancer Res 2019;25:1063-9.

(English Language Editor: J. Jones) 